\title{
Characterization of Humidity Sensing of Polymeric Graphene- Quantum-Dots Composites Incorporated with Silver Nanowires
}

\author{
Lam Minh Long ${ }^{1}$, Nguyen Nang Dinh ${ }^{1, *}$ \\ Hoang Thi Thu' ${ }^{2}$, Huynh Tri Phong ${ }^{2}$, Tran Quang Trung ${ }^{2}$ \\ ${ }^{1}$ University of Engineering and Technology, Vietnam National University, Hanoi \\ 144 Xuan Thuy, Hanoi, Vietnam \\ ${ }^{2}$ University of Natural Science, Vietnam National University, Ho Chi Minh City \\ 227 Nguyen Van Cu Road, District 5, Ho Chi Minh City
}

Received 07 August 2017

Accepted 19 September 2017

\begin{abstract}
Graphene quantum dots (GQDs) were synthesized and incorporated with polyethylenedioxythiophene:poly(4-styrenesulfonate) (PEDOT:PSS), Ag nanowires (AgNWs) to form a composite that can be used for enhancement of relative humidity (RH\%) sensing. The composite films contained bulk heterojunctions of AgNW/GQD and AgNW/PEDOT:PSS. The sensors made from the composites responded well to relative humidity in a range from $10 \%$ to $50 \%$ at room temperature. With an AgNWs content ranging from $0.2 \mathrm{wt} . \%$ to $0.4 \mathrm{wt} . \%$ and 0.6 wt.\%, the sensitivity of the relative humidity sensing devices based on AgNWs-doped GQDs+PEDOT:PSS composites was increased from 5.5\% to $6.5 \%$ and $15.2 \%$, respectively. The response time of the composite sensors was much improved due to AgNWs doping in the composites. For the 0.6 wt.\% AgNWs-doped GQDs+PEDOT:PSS films, the best value of the recovery time was found to be of $30 \mathrm{~s}$.
\end{abstract}

Keyword: Graphene quantum dots (GQDs), Ag-nanowires (AgNWs), nanocomposite, humidity sensing.

\section{Introduction}

Since graphene was discovered, isolated and characterized in 2004 by Geim and Novoselov [1], numerous scientific works have been increasingly done on the application of graphene. This is because graphene possesses many excellent electrical properties, since it is an allotrope of carbon with a structure of a single two-dimensional (2D) layer of $\mathrm{sp}^{2}$ hybridized carbon atoms. Graphene quantum dots (GQDs), as seen in [2,3], are a kind of OD material made from small pieces of graphene. GQDs exhibit new phenomena due to quantum confinement and edge effects, which are similar to semiconducting QDs [4]. It is known that conducting polymers with conjugated backbone and

\footnotetext{
*Corresponding author. Tel.: 84- 904158300.

Email: dinhnn@vnu.edu.vn

https//doi.org/ 10.25073/2588-1124/vnumap.4216
} 
controlled electron characteristics represent promising components of organic-inorganic composites [5]. Various nanocomposite films consisting of conducting polymers mixed with carbon nanotubes (CNT) as an active material have been prepared for application in gas film sensors. Among the conducting polymers, poly(3,4-ethylenedioxythiophene) abbreviated hereafter as PEDOT is of particular interest owing to its remarkable optical, electrical, and electrochemical properties. To obtain PEDOT-based films deposited on almost any surfaces (e.g., conductive or dielectric, flexible, and polymeric), aqueous dispersion of polymeric complex of PEDOT doped by poly(styrenesulfonate) (PSS) is often used. A polymeric anion PSS acts simultaneously as an acid dopant and an anionic surfactant which stabilizes the dispersion of the polymer [6-8]. High enough processing ability and conductivity of the polymer complex PEDOT:PSS make it be one of the most promising conducting polymers. It has been shown that adsorption of gas molecules such as $\mathrm{CO}$ [9] and $\mathrm{NH}_{3}$ [10], as well as vapors of organic solvents [8] or water molecules [11, 12], can strongly affect different physical characteristics of PEDOT:PSS and the relevant composites. This suggests some ground for employing PEDOT:PSS in gas-sensing devices. Olenych et al. [13] used hybrid composites based on polyethylenedioxythiophene:poly(4-styrenesulfonate) (PEDOT :PSS)-porous silicon-CNT for preparation and characterization of humidity sensors. The value of the resistance of the hybrid films was as large as $10 \mathrm{M} \Omega$ that may have caused a reduced accuracy in monitoring the resistance change vs. humidity. Recently, GQDs incorporated with PEDOT:PSS and CNT were prepared in a form of a composite for making the humidity sensors [14]. In comparison with devices made from the PEDOT:PSS+CNT composites, the GQDs+PEDOT:PSS+CNT sensors exhibited much better humidity sensing properties. However, the best humidity sensitivity $(\eta)$ of these sensors reached a value around $11 \%$. Xing et al [15] reported that the formation of a nanometer-scale chemically responsive junction (CRJ) within a silver nanowire (AgNW) strongly affected to sensing properties of nanocomposites. Exposure of the CRJ-containing nanowire to ammonia $\left(\mathrm{NH}_{3}\right)$ induced a rapid $(<30$ s) and reversible resistance change that was as large as $\Delta \mathrm{R} / \mathrm{R}_{0}=(+) 138 \%$ in $7 \% \mathrm{NH}_{3}$ and observable down to $500 \mathrm{ppm} \mathrm{NH}$. Exposure to water vapor produced a resistance increase of $\Delta \mathrm{R} / \mathrm{R}_{0}, \mathrm{H}_{2} \mathrm{O}=(+)$ $10-15 \%$ (for $2.3 \%$ water) while nitrogen dioxide $\left(\mathrm{NO}_{2}\right)$ exposure induced a stronger concentrationnormalized resistance decrease of $\Delta \mathrm{R} / \mathrm{R}_{0}, \mathrm{NO}_{2}=(-)$ 10-15\% (for $500 \mathrm{ppm} \mathrm{NO}_{2}$ ). The proposed mechanism of the resistance response for a CRJ, supported by temperature-dependent measurements of the conductivity for CRJs and density functional theory calculations, is that semiconducting p-type $\mathrm{Ag}_{\mathrm{x}} \mathrm{O}$ is formed within the CRJ and the binding of molecules to this $\operatorname{Ag}_{\mathrm{x}} \mathrm{O}$ modulates its electrical resistance.

Thus in the hope to enhance the sensitivity of the humidity sensors made from GQDs+ PEDOT:PSS composite films, AgNWs were embedded. In this work we report results of our investigation on the fabrication of graphene-quantum dots and nanocomposites of GQDs+PEDOT:PSS with additive AgNWs. The humidity-sensing properties of the composite-film sensors were also presented.

\section{Experimental}

\subsection{Preparation of Ag nanowires}

Firstly, $20 \mathrm{ml}$ of ethylene glycol was heated within stirring in a $250 \mathrm{ml}$ Corning- 0215 glass at $70^{\circ} \mathrm{C}$ for $15 \mathrm{~min}$, then $17 \mathrm{mg}$ of $\mathrm{NaCl}$ was added. Raising temperature up to $100^{\circ} \mathrm{C}, 20 \mathrm{mg}$ of $\mathrm{AgNO}_{3}$ was filled into the glass. The reaction between $\mathrm{NaCl}$ and $\mathrm{AgNO}_{3}$ occurred, resulting in formation of opaque $\mathrm{AgCl}$ solution. Ethylene glycol was decomposed in aldehyde that played a role of a catalyst for 
creating $\mathrm{Ag}$ nuclei. The next step, $5 \mathrm{mg}$ of $\mathrm{KBr}$ was added to the glass and heated up to $140{ }^{\circ} \mathrm{C}$ for 10 min, following $300 \mathrm{mg}$ of PVP was filled and raising temperature to $160^{\circ} \mathrm{C}$. The solution temperature was maintained for $15 \mathrm{~min}$. Finally $250 \mathrm{mg}$ of $\mathrm{AgNO}_{3}$ was added into the solution. The last solution was kept at $160^{\circ} \mathrm{C}$ for $30 \mathrm{~min}$ for growing silver nanowires. In the duration of this time one can observe the change of the solution colour from opaque to bright-gray, proving the formation of AgNWs in the solution. After the solution was cooled automatically to room temperature (in $\sim 90$ $\mathrm{min}$ ), the solution was diluted by $80 \mathrm{ml}$ of ethanol and kept for $10 \mathrm{~h}$ to deposit an AgNWs paste. This paste was put into a glass with $350 \mathrm{ml}$ of distilled water for spinning with $6000 \mathrm{rpm}$ for $30 \mathrm{~min}$ to get silver nanowires adhering to the glass walls. This AgNWs paste was removed from the glass and put into other glass with $200 \mathrm{ml}$ of ethanol. By ultrasonic stirring, the AgNWs paste was dispersed completely in $2 \mathrm{~h}$. Finally $100 \mathrm{ml}$ of distilled water was added into the AgNWs + ethanol solution, totally $300 \mathrm{ml}$ of the AgNWs solution was prepared for further studies.

\subsection{Preparation of GQDs and GQDs + PEDOT:PSS $+A g N W$ composites}

Preparation of GQDs and GQDs + PEDOT:PSS was done following procedures described elsewhere [14]. In this study we used GQDs+PEDOT:PSS mixture with a volume ratio 2/1 of 10wt.\% GQDs solution / PEDOT:PSS, further this solution is called as GPA. Next step, to the GPA solution, a small amount of the AgNWs paste was added. Three types of the samples with the abbreviation of GPA1, GPA2 and GPA3 were prepared, respectively by adding $0.2,0.4$ and 0.6 wt.\% into the GPA solution. The AgNWs pastes were dispersed in the GPA solutions by ultrasonic wave for at $65^{\circ} \mathrm{C} 1$ hour. Using spin-coating, the GPA, GPA1, GPA2 and GPA3 solutions were deposited onto glass substrates which were coated by two silver planar electrode arrays with a length (L) of $10 \mathrm{~mm}$ and separated one from the other by a distance (l) of $5 \mathrm{~mm}$ (see Figure 1).

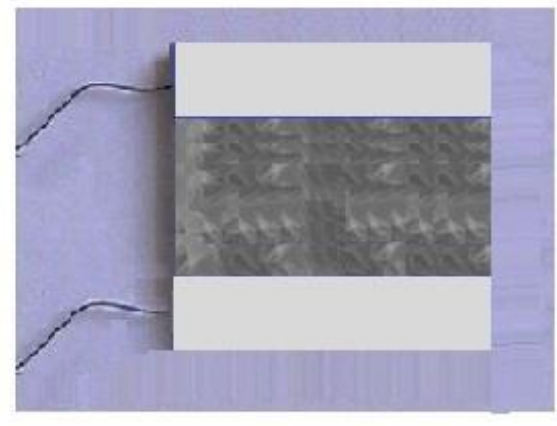

(a)

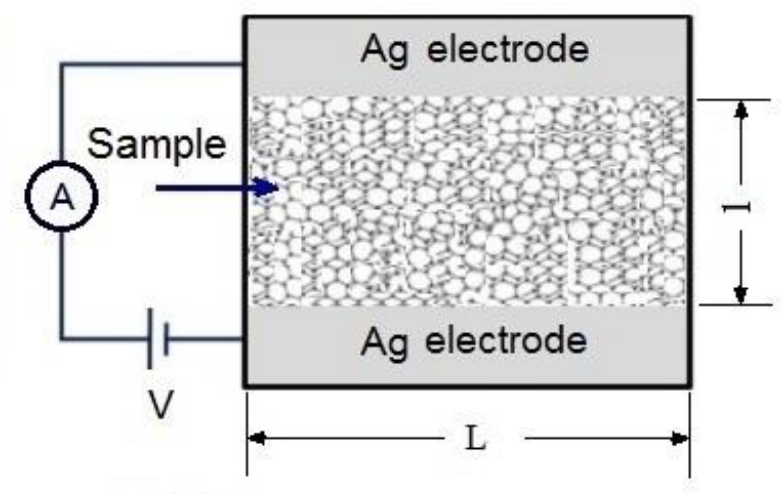

(b)

Figure 1. Image of a humidity sensor made from AgNWs-doped GQDs+PEDOT:PSS composite film (a) and the schematic drawing of the device with the two planar electrodes (b). Humidity change is detected by the change in the current with a constant Dc-bias applied to the two Ag electrodes.

In the spin-coating technique that was used for preparing composite films, the following parameters were chosen: a delay time of $100 \mathrm{~s}$, a rest time of $45 \mathrm{~s}$, a spin speed of $1500 \mathrm{rpm}$, an acceleration of $500 \mathrm{rpm}$, and finally a drying time of $3 \mathrm{~min}$. To dry the composite films, a flow of dried gaseous nitrogen was used for 7 hours. For solidification avoiding the solvents used, the film samples were annealed at $120^{\circ} \mathrm{C}$ for $8 \mathrm{~h}$ in a "SPT-200" vacuum drier. 


\subsection{Characterization techniques}

The thickness of the films was measured on a "Veeco Dektak 6M" stylus profilometer. The size of AgNWs and the surface morphology of the films were characterized by using "Hitachi" Field Emission Scanning Electron Microscopy (FE-SEM). For humidity sensing measurements, the samples were put in a $10 \mathrm{dm}^{3}$-volume chamber, a relative humidity $(\mathrm{RH} \%)$ value could be fixed in a range from $10 \%$ to $70 \%$ by the use of an "EPA-2TH" moisture profilometer (USA). The adsorption process is controlled by insertion of water vapor, while desorption process was done by extraction of the vapor followed by insertion of dry gaseous Ar. The measurement system that was described in [16] consists of an Ar gas tank, gas/vapor hoses and solenoids system, two flow-meters, a bubbler with vapor solution and an airtight test chamber connected with collect-store data DAQ component. The Ar gas played a role as carrier gas, dilution gas and purge gas.

For each sample, the number of measuring cycles was chosen to be at least 10 cycles. The humidity flow taken for measurements was of $\sim 60 \mathrm{sccm} \mathrm{ml} / \mathrm{min}$. The sheet resistance of the samples were measured on a "KEITHLEY 2602" system source meter.

To characterize humidity sensitivity of the composite samples, the devices were placed in a test chamber and device electrodes were connected to electrical feedthroughs.

\section{Results and discussion}

\subsection{Electrical properties and morphology}

To avoid the initial $\mathrm{H}_{2} \mathrm{O}$ vapor in the chamber that strongly affected to the surface resistance of the samples, all the measurements were carried-out at much higher room temperature (namely $50^{\circ} \mathrm{C}$ ). Then humidity sensing measurements were taken on, including two processes: adsorption and desorption by a dried gaseous Ar flow.

The resistivity the samples was determined by using following formula:

$$
\mathrm{R}=\rho \frac{1}{\mathrm{~S}}=\rho \frac{5}{10 \times \mathrm{d}}=\frac{\rho}{2 \mathrm{~d}}
$$
$21 \times d$.

where $\mathrm{d}$ is the film thickness, 1 is the separation distance between two Ag-electrodes, $\mathrm{S}=\mathrm{L} \times \mathrm{d}=$

Thus from the surface resistance one can determine the resistivity ( $\rho$ ) of the films as $\rho=2 R \times d$

Then the conductivity $(\sigma)$ is:

$$
\sigma \sim \frac{1}{\rho}=\frac{1}{2 \mathrm{R} \times \mathrm{d}}
$$

The data of the samples including the AgNWs content, thickness, initial resistance and conductivity are listed in Table 1 . The value of the conductivity of the pure PEDOT:PSS film is $\sim 80$ $\mathrm{S} / \mathrm{cm}$ as reported in [17] that is much larger than the one of the GPA composite films. This proves that the composite films possess a poor concentration of charge carriers. However for materials used in gas sensing monitoring, this fact is an advantage in detecting a small amount of charge carries generated from adsorbed molecules, f. i. $\mathrm{H}_{2} \mathrm{O}$ vapour. 
Table 1. The data of the AgNWs-doped GQDs+PEDOT:PSS composite films used for humidity sensors.

\begin{tabular}{lllll}
\hline $\begin{array}{l}\text { Samples } \\
\text { abbreviation }\end{array}$ & $\begin{array}{l}\text { AgNW content } \\
(\text { wt.\%) }\end{array}$ & $\begin{array}{l}\text { Film thickness, } \mathrm{d} \\
(\mathrm{nm})\end{array}$ & $\begin{array}{l}\text { Resistance at } \\
50^{\circ} \mathrm{C}(\mathrm{M} \Omega)\end{array}$ & $\begin{array}{l}\text { Conductivity } \\
(\mathrm{S} / \mathrm{m})\end{array}$ \\
\hline GPA1 & 0.2 & 450 & 4.56 & 0.024 \\
GPA2 & 0.4 & 460 & 4.24 & 0.026 \\
GPA3 & 0.6 & 480 & 3.88 & 0.027 \\
\hline
\end{tabular}

FE-SEM image of a AgNWs solution (Figure 2a) shows clearly the shape and dimension of the stick-like $\mathrm{Ag}$ wires, as evaluated in this image, the wire size is of $70 \mathrm{~nm}$. Figure $2 \mathrm{~b}$ is a FE-SEM image of the GPA2 film where the AgNWs and GQDs clearly appeared while the conjugate polymer PEDOT:PSS exhibited a transparent matrix. This SEM micrograph also shows that in the composite film there are mainly heterojunctions of the GQD/PEDOT-PSS and AgNW/PEDOT:PSS, whereas both AgNW/GQD junctions are rarely formed.
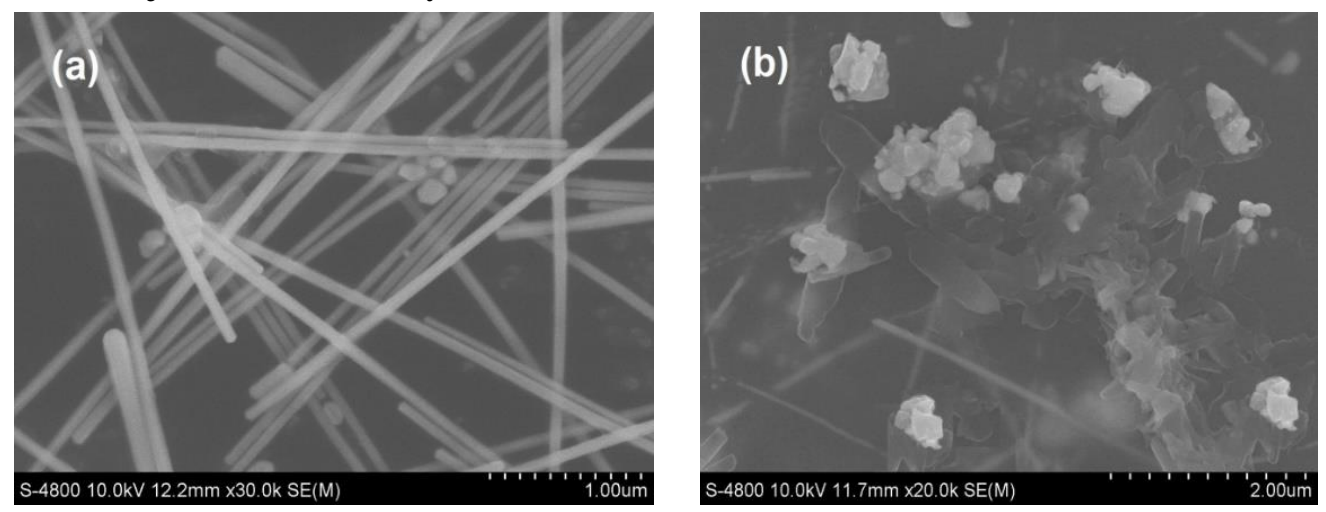

Figure 2. FE-SEM micrograph of an AgNWs containing solution (a) and surface of GPA3 film.

From our experiments, the temperature dependences of the resistance of AgNWs-doped GQDs+PEDOT:PSS composite films was found to be similar to those reported for CNTs-doped GQDs+PEDOT:PSS films [14]. With the increase of temperature, the AgNWs-doped composite exhibited the behavior of a heavily doped semiconductor: the resistance decreased one order in magnitude from the initial values. Indeed, with the AgNWs content of $0.6 \mathrm{wt} . \%$ (GPA3), the resistance of the sensor lowered from $3.88 \mathrm{M} \Omega$ to $400 \mathrm{k} \Omega$ with increase of temperature from room temperature to $80^{\circ} \mathrm{C}$ and maintained a unchanged value of $350 \mathrm{k} \Omega$ under elevated $\left(100\right.$ to $\left.140^{\circ} \mathrm{C}\right)$ operating temperatures. This thermal stability is a desired factor for materials used in sensing applications.

\subsection{Humidity sensing characterization}

In the adsorption process, the humidity flow consisting of $\mathrm{Ar}$ carrier and $\mathrm{H}_{2} \mathrm{O}$ vapor from a bubbler was introduced into the test chamber for an interval of time, following which the change in resistance of the sensors was recorded. In the desorption process, a dried Ar gas flow was inserted in the chamber in order to recover the initial resistance of the GPA films. Through the recovering time dependence of the resistance one can obtain information on the desorption ability of the sensor in the desorption process.

The influence of $\mathrm{H}_{2} \mathrm{O}$ vapour adsorbed on the surface of the sensors was studied by measurements of the humidity dependence of the film resistance in arrange from $\mathrm{RH} 10 \%$ to $\mathrm{RH} 70 \%$. The humidity in 
the chamber was controlled by a humidity standard system "EPA-2TH" (USA). From experimental measurements we have found that the electrical characteristics of our thin-film sensor elements are strongly dependent on the surrounding atmosphere, on humidity in particular. The increase in relative humidity results in significant decrease of the electrical resistance of the GPA composite films, namely GPA1, GPA2 and GPA3 (see Figure 3). At the RH lower 30\%, the resistance of the sensors intensively decreased and reached an almost the same value of $400 \mathrm{k} \Omega$ from $\mathrm{RH}$ larger $50 \%$. This demonstrates that AgNWs-doped GQDs+PEDOT:PSS composite films can be used well for humidity sensing in a range from $\mathrm{RH} 10 \%$ to $\mathrm{RH} 40 \%$. Moreover, in this RH range GRA3 sensor is the most sensitive to humidity, comparing to GRA1 and GRA2.

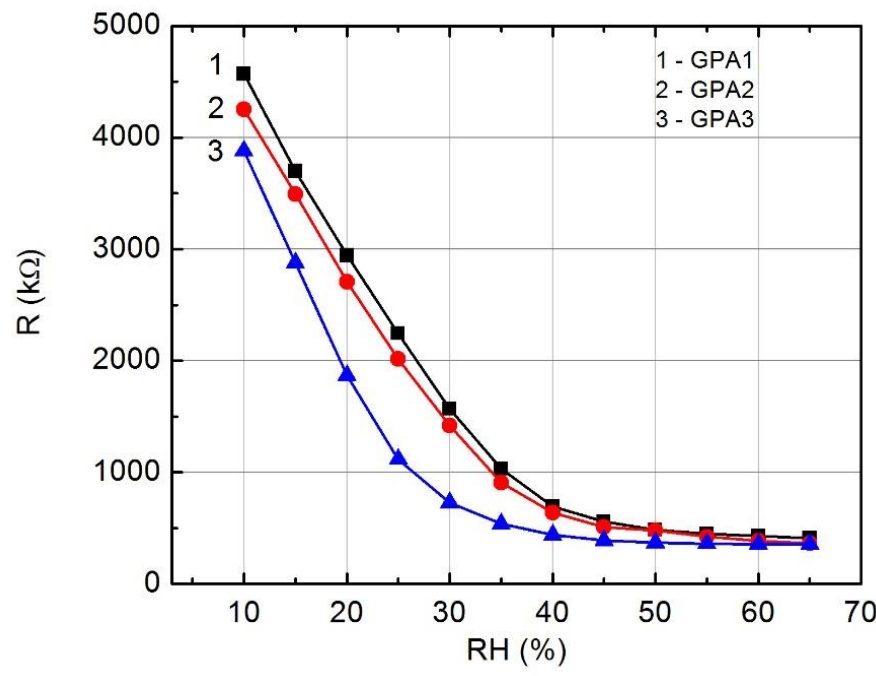

Figure 3. RH\% dependence of the surface resistance of AgNWs-doped GQDs+PEDOT:PSS for three composite films with 0.2 wt.\% (curve "1"), 0.4 wt.\% (curve "2") and 0.6 wt.\% of AgNWs (curve "3").

The humidity dependence of the resistance of the hybrid (or composite) films can be explained by the interaction of water molecules with the surface of the composite, which leads to changing electric parameters of the GQDs. On the other hand, water impurities might induce additional or so called 'secondary' doping of the conjugated polymer PEDOT:PSS. This manifests itself in change of the chain shape to an 'unfolded spiral' and, therefore, stimulates increase in the conductivity [8].

More detailed measurements of the time response of the sensors were carried-out in the conditions of $\mathrm{H}_{2} \mathrm{O}$ vapour insertion and extraction, respectively to the adsorption and desorption processes. Figure 4 demonstrates the results of the measurements for AgNWs-doped GQDs+PEDOT:PSS sensors, i. e. for GPA1, GPA2 and GPA3. From Figure 4 one can see that the best humidity sensitivity was obtained in the sensor made from GPA3 film where the AgNWs content is of $0.6 \mathrm{wt} . \%$. The samples with larger AgNWs contents (namely 0.8 to $1.2 \mathrm{wt} . \%$ ) in the composites were also made, however the sensing to humidity of these composite decreased rapidly. Indeed, in Figure 4 the adsorption and desorption processes of the 0.8 wt.\% AgNWs-doped GQDs+PEDOT:PSS sensor (called as GPA4) were revealed worse than that of the GPA3 sensor (0.6 wt.\% AgNWs). Figure 4 shows that the humidity desorption/adsorption process led respectively to increase/decrease of the resistance of sensors, with results similar to those reported in [18]. 


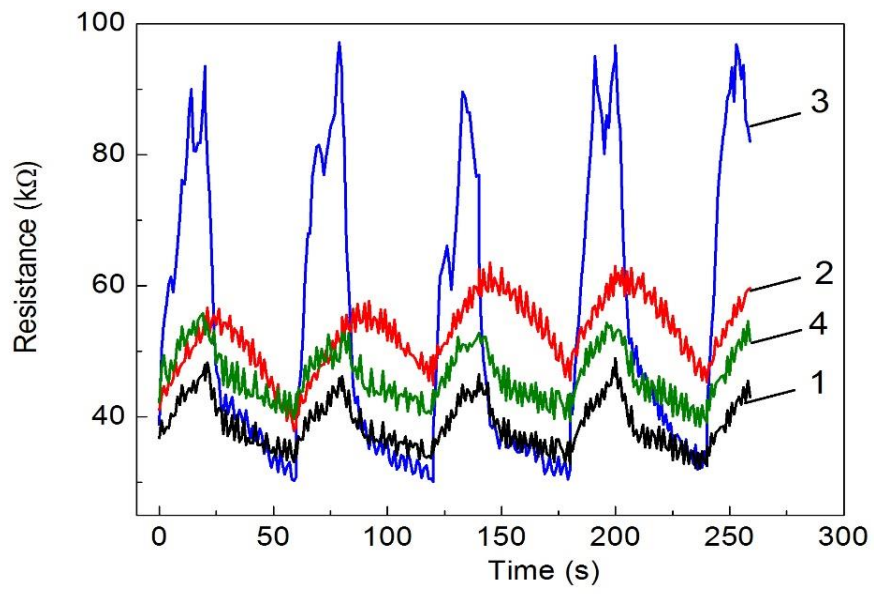

Figure 4. Responses of resistance of the sensors based on AgNWs-doped GQD/PEDOT:PSS films to the pulse of relative humidity (RH 30\%) at room temperature for samples GPA1 (curve "1"), GPA2 (curve "2"), GPA3 (curve "3") and GPA1 (curve "4").

To appreciate better the sensing performance of the GPA composite films used for the sensors, a sensitivity $(\eta)$ of the devices was introduced. It is determined by following equation:

$$
\eta=\frac{\mathrm{R}-\mathrm{R}_{\mathrm{o}}}{\mathrm{R}_{\mathrm{o}}} \%
$$

Figure 5 shows the sensitivity of the GPA3 sensor during 5 cycles of the adsorption and desorption of $\mathrm{H}_{2} \mathrm{O}$ vapour. The absolute magnitude of the sensitivity of the GPA3 calculated by formula (3) reached a value as large as $15.2 \%$. The plots for GPA1 and GPA2 sensors have a shape similar to the one of GPA3 (here they are not presented), however the sensitivity of were smaller, namely $5.5 \%$ and $6.5 \%$, respectively for GPA1 and GPA2. Comparing with the CNT-doped GQDs+PEDOT:PSS film sensor $(\eta \sim 11 \%)$ as reported in [14], the humidity sensing of $0.6 \mathrm{wt} . \%$ AgNWs-doped composite is much larger.

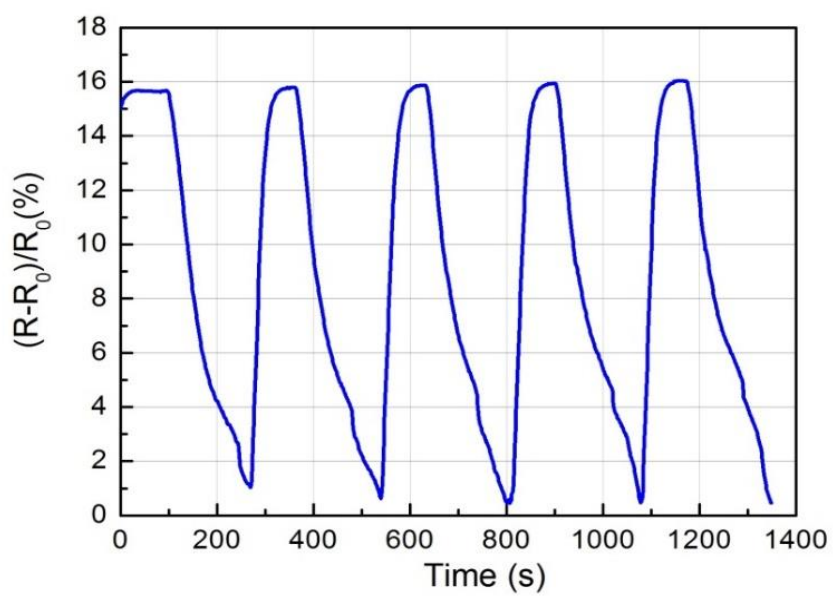

Figure 5. Responses of the sensitivity of the GPA3 sensor to the pulse of relative air humidity (RH30\%) at room temperature. 
In addition, the complete $\mathrm{H}_{2} \mathrm{O}$ molecular desorption on the surface of GPA composites took place at room temperature and atmospheric pressure. One can guess that connecting together individual GPA sheets by AgNWs caused the increase of the mobility of carriers in composite films, consequently leading to higher $\mathrm{H}_{2} \mathrm{O}$ vapor sensing ability of the AgNWs-doped GQDs+PEDOT:PSS composites. Similarly to CNT-doped GQDs+PEDOT:PSS composites, due to the appearance of AgNWs bridges, the number of the sites with high binding energies in GPA sheets decreases, while the number of those with low binding energies increases. Since the $\mathrm{H}_{2} \mathrm{O}$ molecules was mainly adsorbed at the sites with low binding energies, the appearance of AgNWs bridges led to the complete desorption ability of GPA composites.

\section{Conclusion}

The synthesized graphene quantum dots (GQDs) and spin-coated composite thin films of GQDs, PEDOT:PSS and Ag nanowires (GPA) were prepared for characterization of humidity sensing. The composite resistance sensors were made from the GPA films with a simple structure of Ag/composite films/Ag; and these sensors responded well to the humidity change at room temperature and atmospheric pressure. With the AgNWs content increase, from $0.2 \mathrm{wt} . \%$ (GPA1) to $0.4 \mathrm{wt} \%$ (GPA2) and $0.6 \mathrm{wt} \%$ (GPA3), the sensitivity of the humidity sensing devices based on AgNWs-doped graphene quantum dot-PEDOT:PSS composites improved from 5.5\% (GPA1), $6.5 \%$ (GPA2) and 15.2 $\%$ (GPA3), respectively The best response time ( $30 \mathrm{~s})$ was obtained for sensors made from $0.6 \mathrm{wt} . \%$ AgNWs-doped GQDs+PEDOT:PSS composite films.

\section{Acknowledgments}

This research was partially funded by the Vietnam National Foundation for Science and Technology (NAFOSTED) under grant number 103.02-2013.39. The author (LML) expresses grateful thanks to Faculty of Engineering and Nanotechnology, University of Engineering and Technology (VNU Hanoi) and Department of Solid State Physics, University of Science (VNU Ho Chi Minh city) for useful supports in samples preparation and characterization.

\section{References}

[1] K. S. Novoselov, A. K. Geim, S. V. Morozov, D. Jiang, Y. Zhang, S. V. Dubonos, I. V. Grigorieva, A. A. Firsov, (2004-10-22). Electric Field Effect in Atomically Thin Carbon Films. Science (2004) 306 666-669.

[2] J .H. Shen, Y. H. Zhu, X. L. Yang, C. Z. Li, Graphene quantum dots: emergent nanolights for bioimaging, sensors, catalysis and photovoltaic devices, Chem. Commun. (2012) 48 3686-3699.

[3] Z. P. Zhang, J. Zhang, N. Chen, L. T. Qu, Graphene quantum dots: an emerging material for energy-related applications and beyond, Energy Environ. Sci., (2012) 5/10 8869-8890.

[4] L. Li, G. Wu, G. Yang, J. Peng, J. Zhao, J.-J. Zhu, Focusing on luminescent graphene quantum dots: current status and future perspectives, Nanoscale, (2013) 5 4015-4039.

[5] A. J. Heeger, Semiconducting and metallic polymers: the fourth generation of polymeric materials. Synth Metals. (2002) 123 23-42.

[6] O. Pyshkina, A. Kubarko, V. Sergeyev, Poly(3,4-ethylenedioxythiophene): synthesis and properties. Mater. Sci. App. Chem. (2010) 21 51-56.

[7] S.-I. Na, S.-S. Kim, J. Jo, D.-Y. Kim, Efficient and flexible ITO-free organic solar cells using. Adv. Mater. (2008) 20 4061-4068. 
[8] J. Ouyang, Q. Xu, C.-W. Chu, Y. Yang, G. Li, J. Shinar, On the mechanism of conductivity enhancement in poly(3,4-ethylenedioxythiophene): poly(styrenesulfonate) film through solvent treatment, Polymer. (2004) 45 8443-8450.

[9] G. Latessa, F. Brunetti, A. Reale, G. Saggio, A. Di Carlo, Latessa G, Brunetti F, Reale A, Saggio G, Di Carlo A. Piezoresistive behaviour of flexible PEDOT:PSS based sensor, Sensor Actuator B (2009) 139 304-310.

[10] J. Jian, X. Guo, L. Lin, Q. Cai, J. Cheng, J. Li, Gas-sensing characteristics of dielectrophoretically assembled composite film of oxygen plasma-treated SWCNTs and PEDOT/PSS polymer, Sensor Actuator B (2013) 178 279-288.

[11] M. Kuş, S. Okur S, Electrical characterization of PEDOT:PSS beyond humidity saturation. Sensor Actuator B. (2009) 143 177-1781.

[12] K. Saxena, O. Nanda, N. Gupta, P. Kumar and V. K Jain, Humidity sensing response of poly(3,4-ethylene dioxythiophene)-poly(styrene sulphonate) and its nanocomposites. Environmental Science and Engineering: Physics of Semiconductor Devices (2014) p. 417-9.

[13] I. B. Olenych, O. I. Aksimentyeva, L. S. Monastyrskii, Y. Y. Horbenko, L. I. Yarytska, Sensory properties of hybrid composites based on poly(3,4-ethylenedioxythiophene)-porous silicon-carbon nanotubes, Nanoscale Res. Lett. (2015) 10 187-195.

[14] Lam Minh Long, Nguyen Nang Dinh, Tran Quang Trung, Synthesis and Characterization of Polymeric Graphene-Quantum-Dots based Nanocomposites for Humidity Sensing, J. Nanomat. Vol. 2016 (2016) Article ID 5849018, $6 \mathrm{p}$.

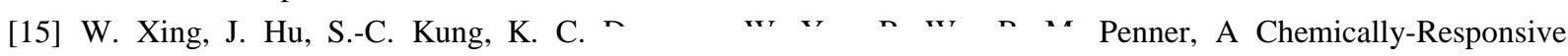
Nanojunction within a Silver Nanowire, Nano Lett. (2012), 12/3 1729-1735.

[16] Tran Quang Trung, Tran My Hoa Huynh, Duc Tai Tong, Tran Van Tam, Nguyen Nang Dinh, Synthesis and application of graphene-silver nanowires composite for ammonia gas sensing, Adv. Nat. Sci.: Nanosci. Nanotechnol. (2013) 4045012 (4p.).

[17] J. Quyang, C.-W. Chu, F.-C. Chen, Q. Xu, Y. Yang, High-Conductivity Poly(3,4ethylenedioxythiophene):Poly(styrene sulfonate) Film and Its Application in Polymer Optoelectronic Devices, Adv. Funct. Mater. (2005) 15 203-208.

[18] J. N. Gavgani, H. S. Dehsari, A. Hasani, M. Mahyari, E. K. Shalamzari, A.Salehi, F. A. Taromi, A room temperature volatile organic compound sensor with enhanced performance, fast response and recovery based on $\mathrm{N}$-doped graphene quantum dots and poly(3,4-ethylenedioxythiophene)-poly(styrenesulfonate) nanocomposite, Royal Soc. of Chem. (2015) 5, 57559-57567. 